\title{
Dune formation in dilute phase pneumatic transport system: PIV \& PTV based analysis
}

\author{
Sumit Jhalani, Atharva Patankar, Ajay Makawana, Manaswita Bose* \\ Department of Energy Science and Engineering, Indian Institute of Technology Bombay, Powai, Mumbai, India - 400076
}

\begin{abstract}
Flow of gas-solid mixture through horizontal conveying section show a large variety of phenomena and is broadly classified into dilute and dense unstable regimes. Different types of instabilities are observed in the dense phase flow and are widely studied in literature; however, clustering instabilities are observed in the very dilute regime of flow with volume fraction $\sim 0.001$. A recent study has shown that regular, stable dune shaped clusters are formed in a small regime of the dilute phase of conveying. The dunes become unstable as the superficial gas velocity is decreased before it finally leads to the dense mode of conveying. The motivation of the current work is to investigate the velocity distribution on the surface of the stable and unstable dunes and thereby understand the cause behind the formation of the dunes in the conveying section. To that end, particle image and tracking velocimetry techniques are employed with the specific objective to determine the volume fraction and the velocity profile of the solid phase on the stable dune surface. A drastic change in the solid fraction within a few particle diameters from the dune surface suggests that PTV is more appropriate in the bulk whereas PIV is suitable for near surface investigation.
\end{abstract}

\section{Introduction}

The flow of suspended solid particles in a gaseous medium through horizontal channel and pipe is widely investigated [1-3], and a large variety of phenomena is reported in the literature. The primary application of the flow of the gas-solid mixture through pipes is pneumatic conveying systems. The design of such systems is in general guided by the classical Zenz plot [4] in which the line pressure drop is plotted as a function of the superficial velocity. The pressure drop shows minima which are known as the saltation point and is typically considered to be the transition point between the dense and the dilute regimes of the transport. Though both dilute [5-8] and dense [9] regimes of the transport systems are well studied in literature; the transition between them is under-examined. It is briefly reported in the literature that the flow becomes unstable near the transition regime and large fluctuation in the line pressure drop is recorded $[1,4,10]$. It was earlier postulated that small unstable clusters form in this regime causing fluctuations in line pressure [1]. In a recent investigation, it is observed that the small regular dune shaped clusters as shown in Figure 1 are formed in the conveying line in the transition regime [11]. The dunes are often stable; however, there is a small region in the flow regime mapping [1] where these dunes are unstable and either their movement in the line or their periodic growth and decay leads to pressure fluctuation [12]. Though the dunes are not common in the context of pneumatic transport systems, they are widely discussed for the atmospheric flows [13]. Quite like the barchans dunes, these clusters are also found to be self-similar in shape [12]. Figure 1b shows that the surface of these clusters is fairly uniform.

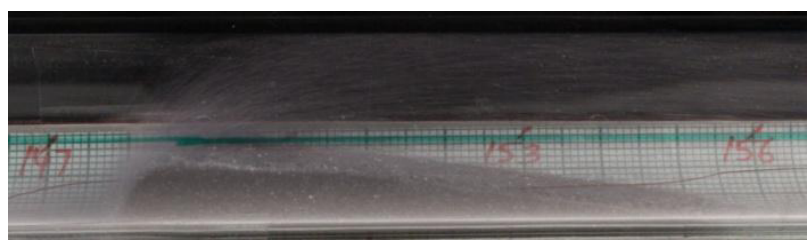

(a)

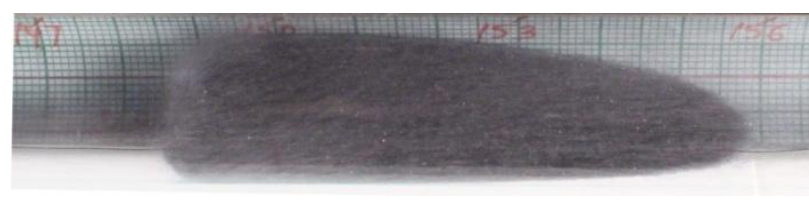

(b)

Fig.1: A typical stable dune in the conveying line captured with Nikon D70 \& AF Zoom-Nikkor 24-85 mm f/2.8-4 D IF lens; (a) The front view; (b) The top view

The formation of either the stable or the unstable dunes in the conveying line is believed to be the result of the particle-particle and fluid-particle interactions, as it is observed for the subaqueous barchan dunes [14-16]. The aim of the current work, therefore, is to develop an

*Corresponding author: manaswita.bose@iitb.ac.in 
understanding of the mechanism of the formation of the dunes in the conveying line.

A careful inspection of the dune shaped structures reveals that immediately above the surface the flow is very dilute, but the solid volume fraction of the dune itself is close to the packed bed limit. In the dilute range the particle tracking velocimetry (PTV) is more appropriate; however in the region very near to the surface, especially close the dune tip where the solid volume fraction is high, the particle image velocimetry is expected to predict the velocities more accurately than the PTV. The specific objective of the current work is to determine the velocity profile of the solid phase on the dune surface using PIV and the PTV techniques as appropriate.

\section{Experimental Method}

\subsection{Experimental set-up}

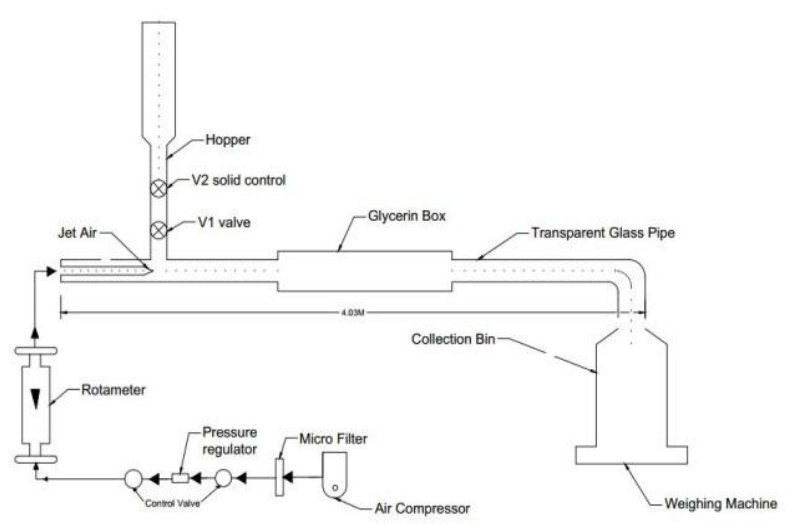

(a)

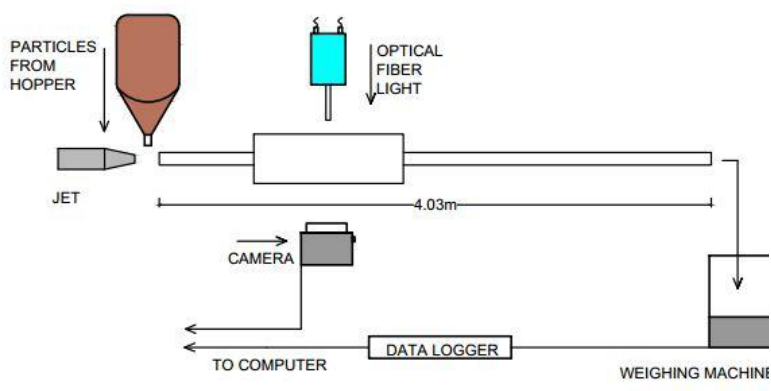

(b)

Fig.2. (a) Schematic of the Experimental set-up; (b) Arrangement of the camera and the illuminator

Figure 2 shows the schematic of the experimental set-up. Particles are fed to the conveying line from a vertically mounted hopper and are conveyed through with the help of a jet ejector pump. The test section is made of borosilicate glass for clear visualisation.
The inner diameter of the pipe is $21.5 \mathrm{~mm}$, and the length of the test section is $500 \mathrm{~mm}$. The particles are collected in a bin placed at the end of the line. The collection bin is placed on a load cell.

The solid mass flow is controlled with the help of two valves and the air flow rate is regulated with the help of a ball valve and pressure regulator. The air flow rate is measured with the help of rotameter and the particle mass flow rate is measured using a load cell. The detail of the experimental set-up and the method are described in [11].

\subsection{High-Speed Imaging}

The motion of the particles is captured using high-speed CMOS camera, Phantom V9.1. The test section is blanketed in a rectangular channel filled with $99 \%$ anhydrous glycerine having the refractive index matched with that of borosilicate glass. The test section is illuminated with fibre-optics light source (Fiber-Lite MH100). The camera is placed at the front of the channel. The rear side of the box is covered with a white translucent paper. The light source is placed at the rear end. Undesired reflections are cut down by blocking the light rays with black cloth. The arrangement of the light source and the camera is shown in Figure $2 \mathrm{~b}$. The shadows of the particles are captured using the highspeed camera at frame rate ranging between 2000 and 3600 fps. Micro-Nikkor $60 \mathrm{~mm} \mathrm{f} / 2.8$ lens is used for capturing the images. The depth of the focus of the lens for all the recording is measured twice the particle diameter. The spatial resolution of the images is 0.06 $\mathrm{mm}$. $(1 \mathrm{~mm}=17$ pixels $)$. The image quality is enhanced with the help of the CSR of the camera. One sample snap shot of the conveying section is shown in Figure $3 \mathrm{a}$. Experiments are also performed with LRS-0532 DPSS laser sheet for brighter illumination and more precise planar measurement. Figure $3 b$ shows a snapshot captured using the green laser sheet.

\subsection{Processing of the Images}

Reference images are captured for the calibration as well as for conversion from pixel to real scale. The images are processed with the standard image processing toolbox of MatLab R2012b for filtering of noise. The images are then processed using the algorithms implemented in PIVLab 1.41. Direct cross-correlation is used for determining the local velocity of the particle phase. Dependence of the velocity on the selection of the window size is checked before the final sampling. The final velocity of the solid phase is obtained by averaging the instantaneous velocities for thousands of frames. Analysis using the particle tracking velocimetry are being carried out with the help of a code developed inhouse [17]. PTV is performed especially in the region far from the dune surface. The results obtained from both methods are being compared. It is worth to note that the imaging, in this case, needs attention as there is a sudden change in the particle volume fraction in the test section and one also has to address the issue of large light scatter 
from the dune surface which forms during the flow. So, a large number of experiments are required even for a single set of flow parameters.

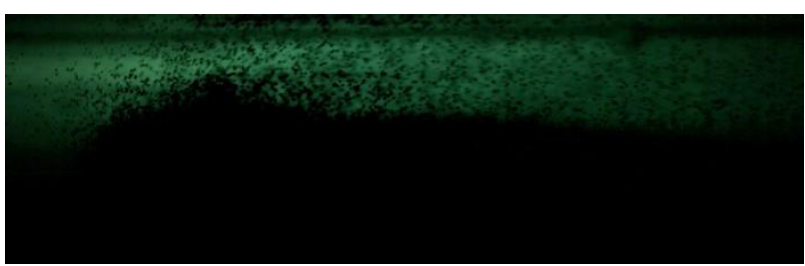

(a)

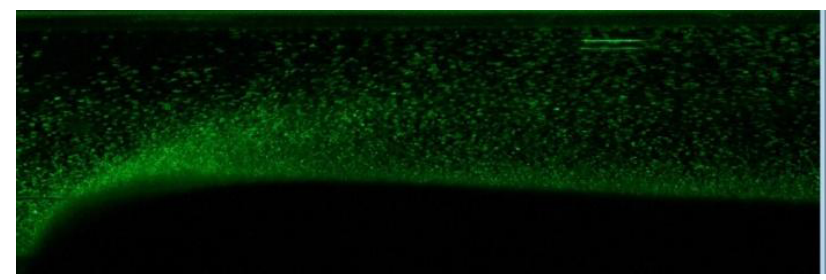

(b)

Fig.3. Snapshot of the Dune using high-speed imaging (a) shadow graphy (b) green laser

\subsection{Methodology}

Table 1 presents the list of parameters for which the experiments are performed. Once the reference image is captured after the camera and the illuminations are set, the air and the solid flow controlling valves are opened and the experiments are initiated. The recordings of the particle motion are started once the dune is completely developed.

Table 1: List of parameters for which the experiments are conducted

\begin{tabular}{|c|c|}
\hline Parameters & Available Range \\
\hline Air flow rate & $6.5 \mathrm{~m}^{3} / \mathrm{hr}$ \\
\hline Box dimensions (LxBxH) & $720 \mathrm{~mm} \times 72 \mathrm{~mm} \times 90 \mathrm{~mm}$ \\
\hline Pipe dimensions (L, D) & $3800 \mathrm{~mm}, 21.5 \mathrm{~mm}$ \\
\hline Air Velocity & $4.91 \mathrm{~m} / \mathrm{sec}$ \\
\hline Particle density & $2500 \mathrm{~kg} / \mathrm{m}^{3}$ \\
\hline Particle diameter (SMD) & $166 \mu \mathrm{m}$ \\
\hline Bulk Volume Fraction & $\sim 0.001$ \\
\hline Mass loading ratio & 3.24 \\
\hline
\end{tabular}

\section{Results}

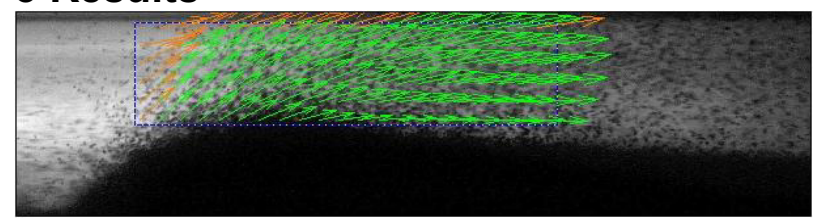

Fig.4. Velocity vectors on the dune surface
The height of the dune changes along its length along the axial direction of the pipe. So the entire frame is divided

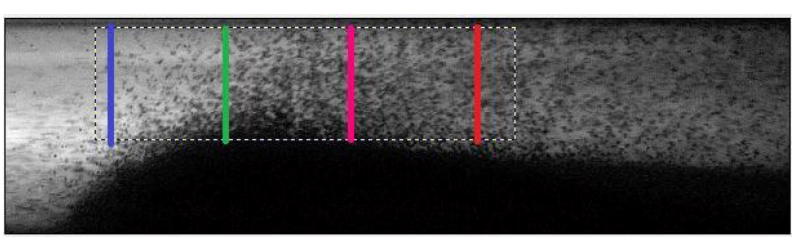

(a)

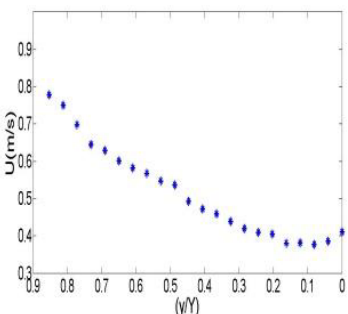

(b)

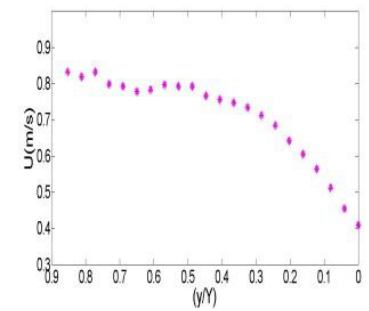

(d)

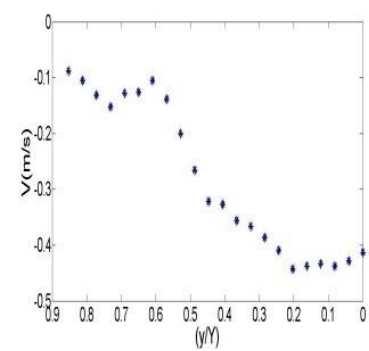

(f)

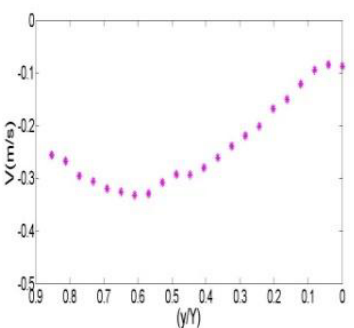

(h)

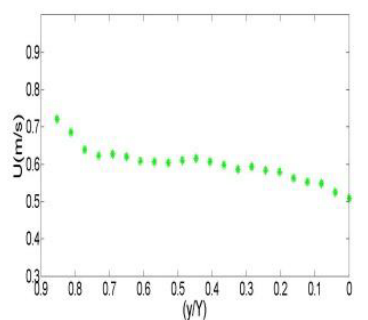

(c)

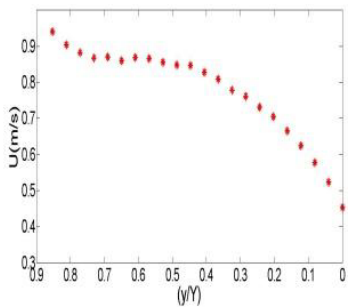

(e)

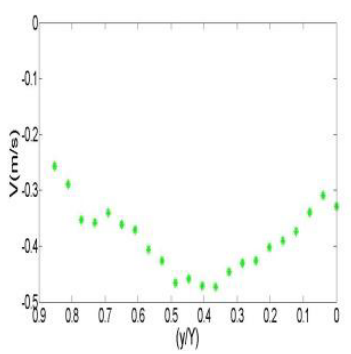

(g)

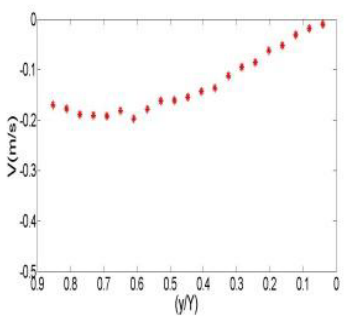

(i)
Fig. 5.(a)The grey scale image of the dune showing the positions on the dune surface where the velocity profiles are obtained; Profiles of the (b) - (e) stream-wise (axial) and (f) (i) cross-stream (gradient direction) components of the particle velocities

in to different regions of interest as shown in Figure 4. The interrogation window and the step size are selected next. The velocity of the particles in each interrogation window at each time instant is then determined. Figure 4 shows the velocity vector plot at one time instant and indicates that there are fluctuations in the particle phase 
velocity. The size of the interrogation window for this case is selected to be $16 \times 16$ pixels with a step size of 8 pixels. The mean profiles of the velocity along the radial direction for different axial positions are finally obtained by averaging the particle velocities over a large number of frames ranging between 20,000 and 25,000. Figure 5a shows a typical grey scale image of a dune with the four different locations at which the velocity profiles are obtained.

The colour code is used for clear representation, e.g., blue is used in Figure $5 \mathrm{~b}$ and $5 \mathrm{f}$ which show the profiles for the stream-wise and the cross-stream components of the particle velocities respectively at the location at the dune tip (marked blue in Figure 5a).

Figures $5 \mathrm{~b}$ to $5 \mathrm{e}$ show the stream-wise components and Figures $5 \mathrm{f}$ to $5 \mathrm{i}$ plot the same for the cross-stream components of the solid phase velocities in the pipe on the dune surface. The stream-wise components have significantly higher magnitude than that of the crossstream components. The particles have smaller velocity at the surface of the dune and larger as they move away from the surface, which is expected. The large slip of the particles at the solid wall is consistent with the observations reported in $[5,6,17]$ for the dilute phase transport. The average solid phase stream-wise velocity (axial) is flatter near the top wall (Figures 5d \& 5e) indicating that the flow is dilute; however, this kind of demarcation is not possible near the dune crest (Figures $5 \mathrm{~b}$ and $5 \mathrm{c}$ ). Near the surface of the dunes, the particles collide with those on the surface resulting in either rebound or rolling over the surface. Sometimes the particles in flight settle on the dune surface losing the momentum to the clusters; whereas at some other times the collisions result in the ejection of particles from the surface of the dunes; however, for the stable dunes, a steady state is maintained. The detailed dynamics of the particles near the dune surface are not reflected in the profiles of the average velocities of the particles. The distribution of the fluctuating velocities near the dune surface needs to be determined for a better insight.

\section{Discussion}

Clustering instability in shape of dunes is observed in the dilute regime of the pneumatic transport system. The formation of the dunes is believed to be the result of interaction between the fluctuating motions of the particles and that of the fluid phase turbulence. To understand the reasons behind the formation of dune shaped clusters in the conveying line, the dynamics of the particles are investigated through PIV and PTV algorithms. The profiles of the solid phase volume fraction and the average velocity are determined at the centre of the pipe. The detailed simulation based on the coupled CFD-DEM algorithms [18] along with appropriate contact models $[19,20]$ need to be carried out for developing more insight into the mechanism of cluster formation. The results of the present experiments can be used as the benchmark for comparing the numerical simulations.

\section{Acknowledgements}

The work is supported by Department of Science and Technology through SR/S3/CE/028/2011 and IITB funding through 15IRINTRN026.

\section{References}

1. Y. Tsuji and Y. Morikawa, Int. J. Multiph. Flow, 8, 329-341, (1982)

2. M. Hubert and H. Kalman, Powder Technol., 134, 156-166, (2003)

3. Y. Zheng and A. Rinoshika, Int. J. Multiph. Flow, 53, 114-123, (2013)

4. C. Crowe, Multiphase Flow Handbook, 4-12. Boca Raton, FL: CRC Press, Taylor and Francis Group, (2006)

5. Y. Tsuji, Y. Morikawa, T. Tanaka, N. Nakatsukasa and N. Nakatani, J. Fluid Mech., 13, 671-684, (1987)

6. S. Lain and M. Sommerfeld, Int. J. Multiph. Flow, 39, 105-120, (2012)

7. D. Kullick, J. Fessler, and J. Eaton, J. Fluid Mech., 277, 109-134, (1994)

8. T. Tanaka and J. Eaton, Phys. Rev Lett., 101, 114502-1 (2008)

9. Y. Tomita and H. Asou, Powder Technol., 196, 14-21, (2009)

10. M. Ochi and K. Ikemori, JSMEB, 21, 10081014, (1978)

11. A. Makwana, A. Patankar, and M. Bose, Part. Sci. Technol., 33, 59-66, (2015)

12. A. Makwana, A. Patankar, and M. Bose, (Manuscript under preparation)

13. B. Andreotti, P. Claudin, and S. Douady, Eur. Phys. J. B, 28, 321-339, (2002)

14. E. Franklin and F. Charru, J. Fluid Mech., 675, 199-222, (2011)

15. F. Charru and M. Franklin, J. Fluid Mech., 694, 131-154, (2012)

16. R. Hout, J. Fluid Mech., 715, 389-423, (2013)

17. A. Deshmukh, V. Vasava, A. Patankar and M. Bose, Powder Technol., 298, 119-129, (2016)

18. Y. Tsuji, T. Kawaguchi and T. Tanaka, Powder Technol., 77, 79-87, (1993)

19. M. J. Jiang, H.-S. Yu and D Harris, Comput. Geotec. 32, 340-357, (2005)

20. M. Jiang, Z. Shen, J. Wang, Comput. Geotec. 65, 147-163, (2015) 